Available online at $\quad$ http://www.jfas.info

\title{
THE ORGANIZATION OF ASSOCIATIVE MEMORY WITH LAMINATION OF
} ELEMENTS

\author{
E. A. Titenko \\ Southwest State University, city Kursk
}

Published online: 08 August 2017

\begin{abstract}
Article contains the description of parallel search in associative memory. Initial operands (words) гесеive a dual impression. The source text is presented in the form of a line or in the form of a matrix. The sample is presented by a two-dimensional form. It consists in splitting a sample into even and odd character sets. Addition of the second coordinate for a sample allows to execute the alternating development of symbols and provides the anticipatory analysis of symbols of a sample of rather next symbols. This feature of the form of a sample allows to process each character set parallely. Processing of character sets is carried out on bit cutoffs in the associative memory at the same time. For the hardware support of steps of retrieval operation the original structure of the associative memory is offered. The structure contains new communications between storage cells, two retrieval registers for storage of character sets and original a circuitry element for foliation of characters on sets.
\end{abstract}

Keywords: search in a sample, matrix, associative memory, stratification of elements.

\section{INTRODUCTION}

It is known that in five-level classification of calculations [1] the processing of symbolical information (PSI) takes the defining place in processes and computing means of support of decision-making. This class of calculations, including, is focused on application of the basic scheme of decision-making by the person "condition-action". Formal reflection of this scheme is the special system of rules (the system of produktion), and basic computing operations search in a sample and modification of structure of data [2].

Author Correspondence, e-mail: author@gmail.com

doi: http://dx.doi.org/10.4314/jfas.v9i2s.87 
Standard processes of search in a sample and modification of data are standardly described over one-dimensional objects - lines, lists, trees, etc. At the same time the interpretation of the processed objects (a word sample, a word modifier, an initial word (text)) only as onedimensional objects leads to unproductive expenses of time, especially when processing large volumes of irregular data, owing to consecutive case-by-case comparison of symbols [3].

\section{The associative memory with lamination of elements}

In operation implementation of parallel search for production systems on the basis of the associative memory consisting of $N$ cells and $\mathrm{m}$ of bit cutoffs (fig. 1) is offered. The temporal scoring in case of the associative search is reached due to parallel processing of all elements of a bit cutoff. As a result time of the associative search is defined as the $\mathrm{T}(\mathrm{m})$ function.

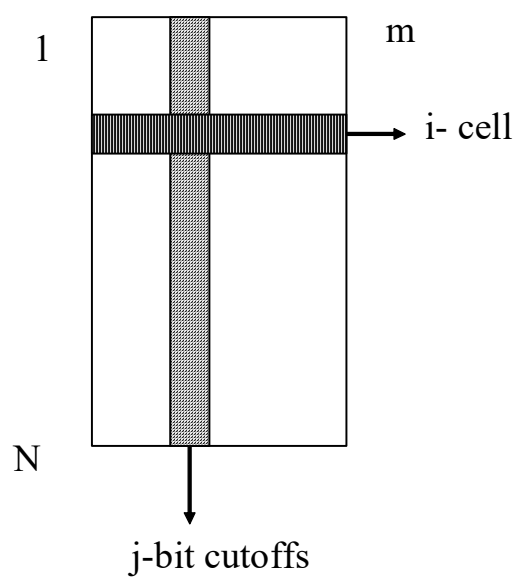

Fig.1. The scheme of access to store elements in associative memory

The associative search on a sample consists of m-1 of the cycles including a step of parallel comparing on bit cutoffs and a step of shift of the initial word on 1 character towards an initial line item. The step of shift is necessary for execution of search in the following line items of the initial word. The principal lack of the associative search on a sample - impossibility "peeping forward". Absence of additional information on a line item of possible entrance does not allow to make the left shift more, than on 1 character that is not always acceptable.

The entity of parallel associative search with "peeping forward" consists in special representation of both operands of retrieval operation. The first operand is the initial word $\mathrm{S}$ length of $\mathrm{N} \times \mathrm{m}$ of characters. It is represented a matrix with serial communications of elements between lines and columns. The second operand is a word - O sample. For parallel processing it is laminated on 2 character sets in all even and odd positions respectively $-\mathrm{O}^{\text {even }}$ and $\mathrm{O}^{\text {odd }}$. Artificial emergence of the second coordinate for a linear (one-dimensional) object 
allows to execute the alternating development of symbols forward - down. The property of an arrangement only of one symbol from $\mathrm{O}$ sample on each of two coordinates allows "to glance forward" on 2 symbols rather current and to conduct parallel search in each coordinate. Demonstrations diagonal-chess (two-dimensional and three-dimensional) representations of a sample are shown in fig. 2. Here development of symbols $\mathrm{O}$ at first forward on the coordinate of $\mathrm{X}$, then on $\mathrm{Y}$ coordinate is by default accepted down.

\begin{tabular}{|c|c|c|c|c|c|}
\hline 0 & $\rightarrow$ & & & & \\
\hline$\downarrow$ & $\mathrm{a} 0$ & a1 & & & \\
\hline & & a2 & a3 & & \\
\hline & & & a4 & a5 & \\
\hline & & & & a6 & a7 \\
\hline & & & & & a8 \\
\hline
\end{tabular}

Fig.2. Two-dimensional representation of a sample $\mathrm{O}$ and his shuffling

Such foliation of characters of a sample of $\mathrm{O}$ allows to increase the frequency of comparing twice. At the same time "peeping forward" is carried out due to implementable shift on a matrix on two coordinates at the same time.

As a result, the organization of associative memory is proposed (Fig. 3) with a twodimensional representation of the original word and the sample word for parallel search. 


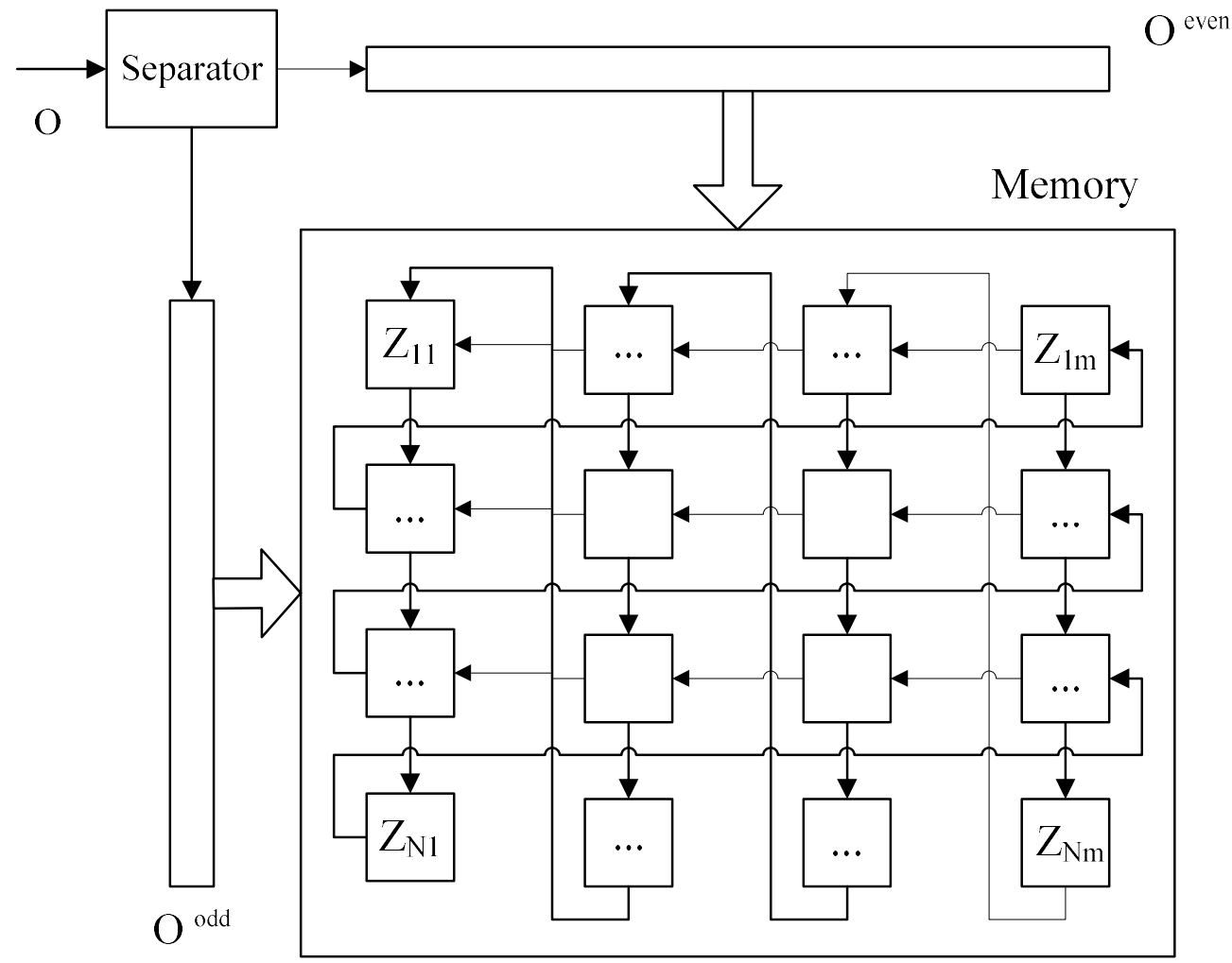

Fig.3. The organization of associative search with lamination of cells

The realization of associative search with stratification of cells is in parallel enabled on two character sets of a sample in even positions and odd positions of a sample of $\mathrm{O}^{\text {even }} \mathrm{O}^{\text {odd }}$ respectively. Introduction of the second coordinate to representation $\mathrm{O}$ allows to stop ahead of schedule search at detection of negative result on one of character sets. The structure of the hoarder (Memory) contains the original circuitry Separator element which is realizing function of lamination of elemet on sets of even and odd characters. Separator contains a binary stage counter which output is address for the demultiplexer. The outputs of the demultiplexer are the outputs of the Separator Element.

\section{CONCLUSION}

In this paper, a hardware-oriented approach has been created for parallel associative searches based on a pattern.Approach differs in special two-dimensional representation of a sample. Two-dimensional representation is created to sets of $b$ of even and odd characters. This additional information algorithmically maintains property "peeping forward" by search due to implementation of shift in the two-dimensional drive on two coordinates. 


\section{REFERENCES}

1. Wa, B.U. The computer for processing of symbolical information / Louray M.B., Gotsze Li.//TIIER. 1989. t.77, N 4. Page 5-40.

2. AlTurani, I.M. Architecture and hardware Solutions Symbolic Information Processing/. I.M. AlTurani, N.I. Zanoon, E.A. Titenko//European Journal of Scientific Research, Volume 68, Issue 3/January, 2012 P.291-296.

3. Titenko, E.A. Organization of the reconfigurable multiprocessor of a data stream / E.A. Titenko//Messenger of computer and information technologies. 2012. No. 2. - Page 24-29.

\section{How to cite this article:}

Titenko E A. The organization of associative memory with lamination of elements. J. Fundam. Appl. Sci., 2017, 9(2S), 1134-1138. 